\title{
Silicon micromachined ultrasonic immersion transducers
}

\author{
H. T. Soh, I. Ladabaum, ${ }^{\text {a) }}$ A. Atalar, ${ }^{\text {b) }}$ C. F. Quate, and B. T. Khuri-Yakub
}

E. L. Ginzton Laboratory, Stanford University, Stanford, California 94305

(Received 15 July 1996; accepted for publication 1 October 1996)

\begin{abstract}
Broadband transmission of ultrasound in water using capacitive, micromachined transducers is reported. Transmission experiments using the same pair of devices at 4,6 , and $8 \mathrm{MHz}$ with a signal-to-noise ratio greater than $48 \mathrm{~dB}$ are presented. Transmission is observed from 1 to $20 \mathrm{MHz}$. Better receiving electronics are necessary to demonstrate operation beyond this range. Furthermore, the same pair of transducers is operated at resonance to demonstrate ultrasound transmission in air at $6 \mathrm{MHz}$. The versatile transducers are made using silicon surface micromachining techniques. Computer simulations confirm the experimental results and are used to show that this technology promises to yield immersion transducers that are competitive with piezoelectric devices in terms of performance, enabling systems with $130 \mathrm{~dB}$ dynamic range. The advantage of the micromachined transducers is that they can be operated in high-temperature environments and that arrays can be fabricated at lower cost. (C) 1996 American Institute of Physics. [S0003-6951(96)00250-1]
\end{abstract}

The propagation of ultrasound in solids and fluids is a physically and mathematically rich field of study ${ }^{1}$ with many practical applications. Ultrasound is widely used in medical imaging and nondestructive evaluation. It is also applied in gas flow metering, industrial cleaning procedures, soldering, position sensing, wafer temperature sensing, and other areas. In order to harness the practical potential of ultrasound, transducers capable of emitting and detecting ultrasound are necessary. Currently, the vast majority of ultrasound transducers are fabricated using piezoelectric crystals and composites. ${ }^{2}$ However, piezoelectric transducers have drawbacks that motivate our novel approach to transducer design. Capacitive acoustic devices have existed for many decades ${ }^{3}$ while capacitive ultrasonic devices are a more recent invention. ${ }^{4,5}$ None of the early ultrasonic devices were fully micromachined, nor was their operation well understood. Recently, micromachined ultrasonic transducers (MUTs) have been shown to be superior to piezoelectric transducers in the generation and detection of ultrasound in air. ${ }^{6,7}$ In this letter, we report the first demonstration of ultrasound generation and reception in water using MUTs. We also introduce simulation results, which imply that MUTs may be a preferable alternative to piezoelectric transducers.

Piezoelectric transducers are problematic for two reasons. First, they require special matching layers in order to couple ultrasound into a liquid or gas medium and these layers need to be manufactured to tight mechanical tolerances. Theoretically interesting designs, such as complex arrays, are limited to realizable configurations, and the cost of manufacturing can be high. Second, piezoelectrics depole at relatively low temperatures $\left(\sim 80^{\circ} \mathrm{C}\right)$, while transducers capable of operating above this temperature are highly desirable.

The design of an immersion MUT element is shown in Fig. 1. A transducer is made of multiple elements, as shown in the scanning electron microscopy (SEM) of Fig. 2. A transducer is efficient if the energy associated with its sur-

${ }^{a)}$ Electronic mail: igal@macro.stanford.edu

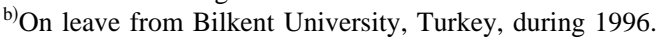

face motion when unloaded is low compared to the energy associated with the surface motion when loaded by the medium of interest (e.g., water). Thus, the surface of motion should be associated with a light structure. The structure can be made resonant to further enhance energy transfer. Our thin resonant membrane fits these criteria. For a thin membrane to resonate in the $\mathrm{MHz}$ range, it must be very stiff, which consequently requires a large activation force. Large Coulombic forces are realized when an electrical potential is applied across a small gap. Thus, a thin metalized membrane separated from a conducting backplate by a small gap is a critical feature of our design. Because detection entails measuring the fractional change of the MUT capacitance when ultrasonic waves impinge on it, a small gap is also desirable in order to maximize detection sensitivity. Micromachining techniques are a good fit to the design requirements of Coulombic ultrasonic transducers.

Figure 3 shows the result of transmission through air at the $6 \mathrm{MHz}$ air-loaded resonant frequency of the membrane. A 10 cycle $16 \mathrm{~V}$ tone burst was applied to the transmitter. The MUTs used in the transmission experiment have an area of $1 \mathrm{~cm}^{2}$. Because the fabrication process is specifically designed to yield vacuum sealed cavities, the same devices can be used for both air and water transmission. Figure 4 shows the results of three water transmission experiments with excitation of 4 cycle $16 \mathrm{~V}$ tone bursts. In Figs. 3 and 4, electromagnetic feedthrough is followed by the acoustic signal, and in the case of water, many subsequent echoes. The echoes indicate that the MUTs are not well matched to the medium. This result is expected because the transducers are optimized for air operation, not water immersion. The geometry of the device (gap thickness, membrane thickness, and radius) and the bias voltage of the device can be modified to
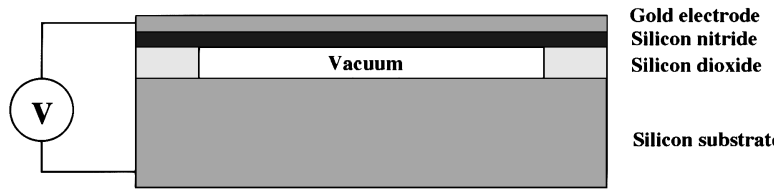

FIG. 1. Schematic of one element of a MUT in cross section. 


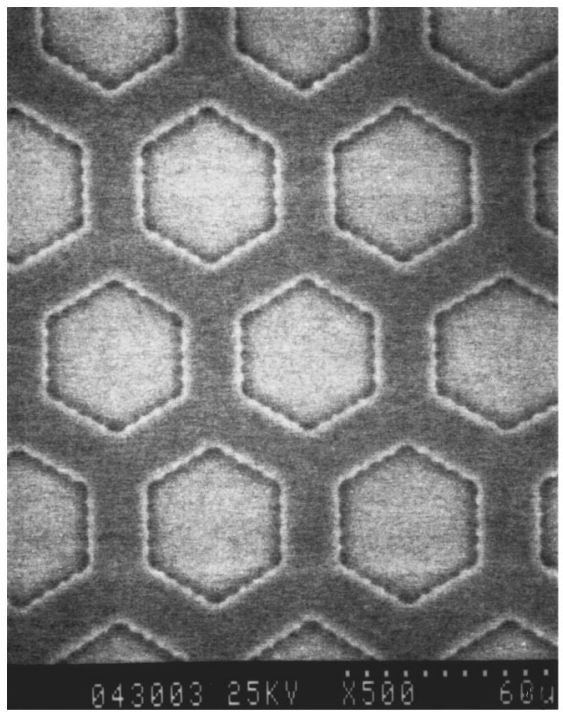

FIG. 2. SEM micrograph of a MUT (top view).

optimize the transducers for water operation; second generation sealed MUTs specifically designed for water immersion are in fabrication. Nevertheless, a signal-to-noise ratio in excess of $48 \mathrm{~dB}$ is evident in the transmission figures. A further reduction in efficiency stems from the fact that a MUT is comprised of thousands of active elements joined by a supporting structure. The inactive supporting structure is responsible for reduced efficiency, so its surface area should be minimized in future designs. Transmission signals are obtained from $1 \mathrm{MHz}$ to frequencies as high as $20 \mathrm{MHz}$, with the bandwidth being limited by the receiving electronics. The electrical tuning of the MUT increases its efficiency at the expense of the bandwidth, and the specific point of operation depends on system requirements. These experiments evidence that capacitive micromachined transducers can operate in a fluid medium with a very large bandwidth.

Computer simulations based on the theory of equivalent circuits of vibrating plates ${ }^{3}$ give reasonable agreement with the two-way insertion loss observed in transmission experiments (see Fig. 5). Computer simulations using optimized design parameters indicate that immersion MUTs can be as sensitive in the receive mode as piezoelectric transducers. The thermal noise behavior of a system can be predicted with an adequate understanding of the dissipative elements of the system (the real part of the impedance). ${ }^{8,9}$ The real part of a MUT's impedance can be manipulated by varying

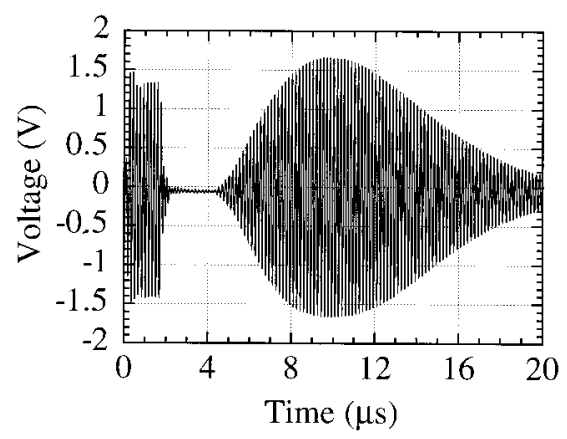

FIG. 3. Transmission in air with a $6 \mathrm{MHz} 10$ cycle burst. (a)

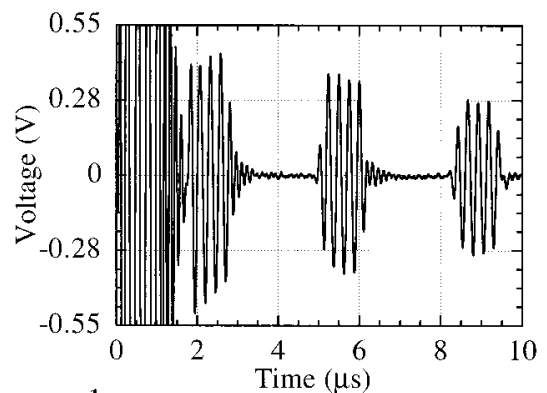

(b)

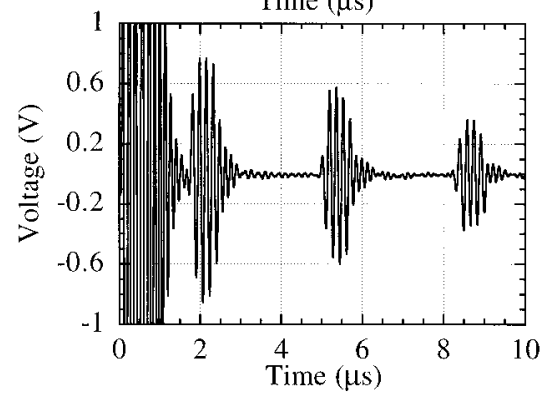

(c)

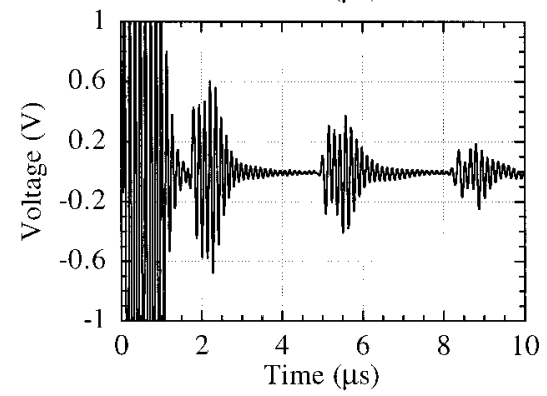

FIG. 4. (a) Transmission in water with a $4 \mathrm{MHz} 4$ cycle burst. (b) Transmission in water with a $6 \mathrm{MHz} 4$ cycle burst. (c) Transmission in water with an $8 \mathrm{MHz} 4$ cycle burst.

the number of elements that are connected in parallel to form the MUT, so it can be engineered to match the optimal source resistance specified for the noise performance of the receiving electronics. Thus, micromachining allows not only for the relatively facile fabrication of MUT arrays, but also for the optimization of the noise performance of the array elements. The one-way insertion loss calculated for an optimized immersion MUT is presented in Fig. 6. Such simulations imply that with optimized parameters a $130 \mathrm{~dB}$ dy-

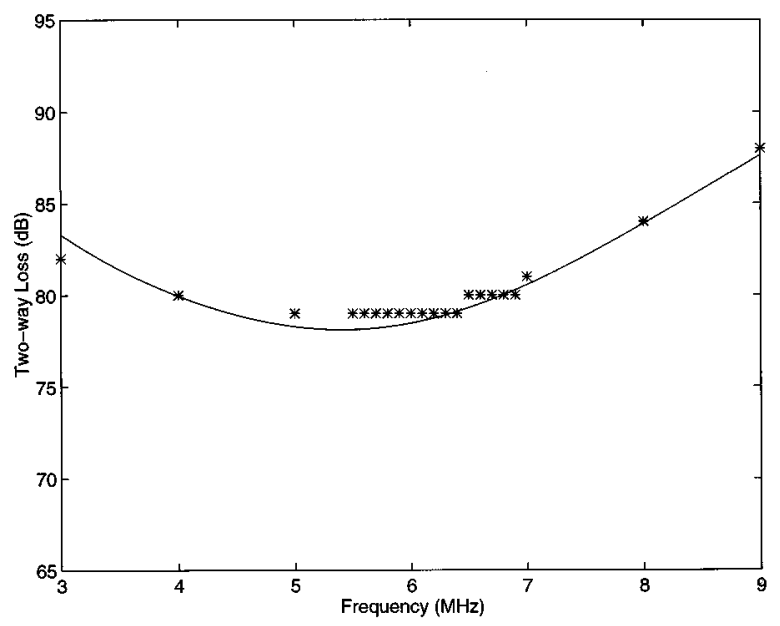

FIG. 5. Insertion loss (theory [-] and experiment [*]) for water transmission. 


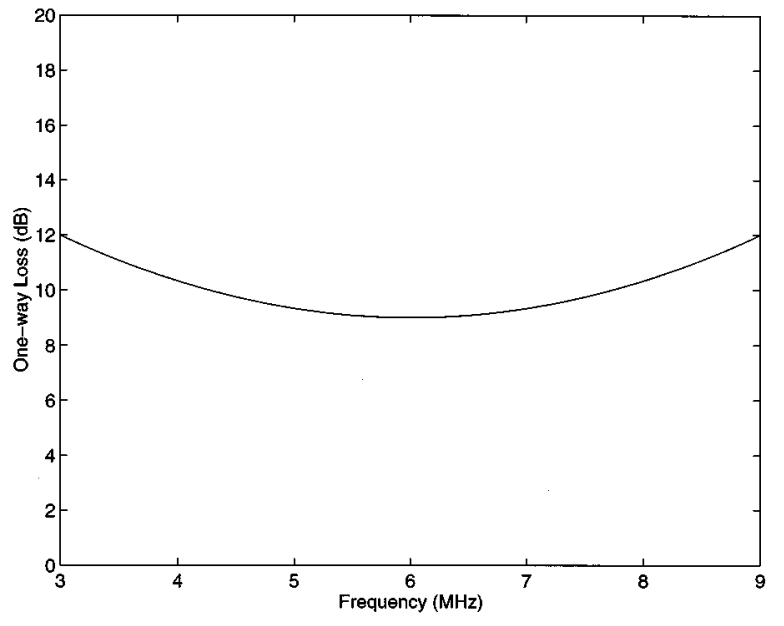

FIG. 6. Theoretical insertion loss for an optimized immersion MUT.

namic range is feasible in a $3 \mathrm{MHz}$ bandwidth. Further restriction of the bandwidth increases the dynamic range.

In conclusion, we have demonstrated that a pair of immersion MUTs can transmit ultrasound in water from 1 to 20 $\mathrm{MHz}$. These transducers were also able to send and receive airborne ultrasound at $6 \mathrm{MHz}$. Simulations predict a dynamic range of $130 \mathrm{~dB}$ in water. The immersion MUTs promise to be a viable alternative to piezoelectric transducers. They have a broad bandwidth and a wide temperature tolerance. Furthermore, microfabrication enables the fabrication of complex array geometries at low cost.

This work was made possible by a grant from the U.S. Office of Naval Research. The authors would also like to acknowledge the helpful contributions of Dr. Mark McCord, Tom Carver, and the Stanford Nanofabrication Facility supported by the NSF.

${ }^{1}$ Lord Rayleigh, The Theory of Sound (Macmillan, London, 1894).

${ }^{2}$ G. S. Kino, Acoustic Waves: Devices Imaging, and Analog Signal Processing (Prentice Hall, Englewood Cliffs, 1987).

${ }^{3}$ W. P. Mason, Electromechanical Transducers and Wave Filters (Van Nostrand, New York, 1942).

${ }^{4}$ J. H. Cantrell and J. S. Heyman, Rev. Sci. Instrum. 50, 31 (1979)

${ }^{5}$ D. W. Schindel and D. A. Hutchins, Proceedings of the 1991 Ultrasonics Symposium, 301 (1991).

${ }^{6}$ I. Ladabaum, D. Spoliansky, and B. T. Khuri-Yakub, Appl. Phys. Lett. 68, 7 (1996).

${ }^{7}$ M. I. Haller, Ph.D. thesis, Stanford University, 1994.

${ }^{8}$ T. B. Gabrielson, IEEE Trans. Electron Devices 40, 903 (1993).

${ }^{9}$ H. B. Callen and T. A. Welton, Phys. Rev. 83, 34 (1951). 